\title{
Failure Analysis of Crimp Connectors
}

\author{
P.T. Vianco, A.C. Kilgo, R. Grant \\ Sandia National Laboratories, Albuquerque, NM 87185
}

Connectors are critical components in today's high-reliability electronics. As connectors decrease in size, the solder joints are being replaced with crimp connections that are easier to make at reduced dimensions. The long-term reliability of the crimp connection rests upon the durability of the cold weld that is formed between the mutual surfaces. The failure of the surfaces to form an integral bond can lead to increased noise on low-voltage (digital) circuits, increased impedance for high frequency circuits, intermittent signal transmissions, or a complete failure of the conduction path.

The integrity of the cold weld that is formed between the contact surface and the pin surface is dependent upon three general conditions: (a) cleanliness of the surfaces, (b) the ability of the contact and pin to deform under the crimping action, and (c) the cold weld withstanding the force of the elastic rebound after removal of the crimping load.

A case study is presented whereby the integrity of the crimp connections was examined in a miniature connector. Energy dispersive $\mathrm{x}$-ray analysis (EDXA) confirmed the contact material to be beryllium-copper ( $\mathrm{Be}-\mathrm{Cu})$ alloy that had been electroplated with a nickel (Ni) layer followed by a gold $\mathrm{Au}$ ) finish. The wires were of stranded copper $(\mathrm{Cu})$ conductor with each strand having an electroplated silver (Ag) finish.

The high magnification images in Fig. 1 show one of the crimp joints. The red arrows in Fig. 1a indicate a gap between the wire strands and the contact surfaces. The optical micrograph in Fig. 1b shows a periodicity of two fracture morphologies. One morphology was separation between the Au plating and the Ag plating on the contact and stranded wire, respectively (green arrow). The second morphology was separation between the $\mathrm{Ag}$ finish of the wire and bare $\mathrm{Be}-\mathrm{Cu}$ of the contact (orange arrow). In the case of the first failure site, it is recognized that $\mathrm{Au}$ and $\mathrm{Ag}$ surfaces are susceptible to contamination from exposure to the air environment. Such contamination, whether on one or both surfaces, can interfere with the formation of the cold-weld bond.

In the second case (orange arrow), the damage originated from the forming process used to make the crimp structure. There was Au as well as Ni plating layers within those near-surface cracks that confirmed that the damage occurred prior to the electroplating process as shown in Fig. 2. The pressure of the crimping process loosened or fragmented the damaged segments. Then, upon the elastic rebound, those fragments separated from the contact surface and were lost during the metallographic cross section preparation process.

\section{Acknowledgement}

Sandia National Laboratories is a multi-program laboratory managed and operated by Sandia Corporation, a wholly owned subsidiary of Lockheed Martin Corporation, for the U.S. Department of Energy's National Nuclear Security Administration under Contract DE-AC04-94AL85000. 


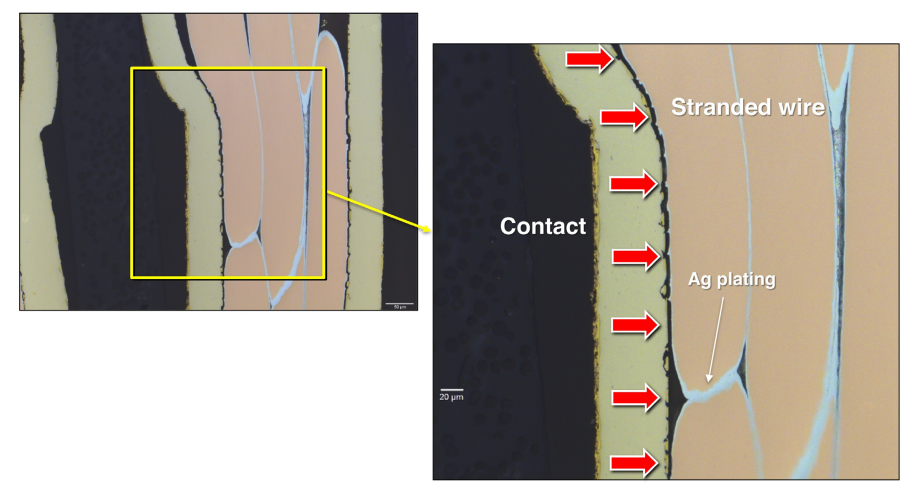

(a)

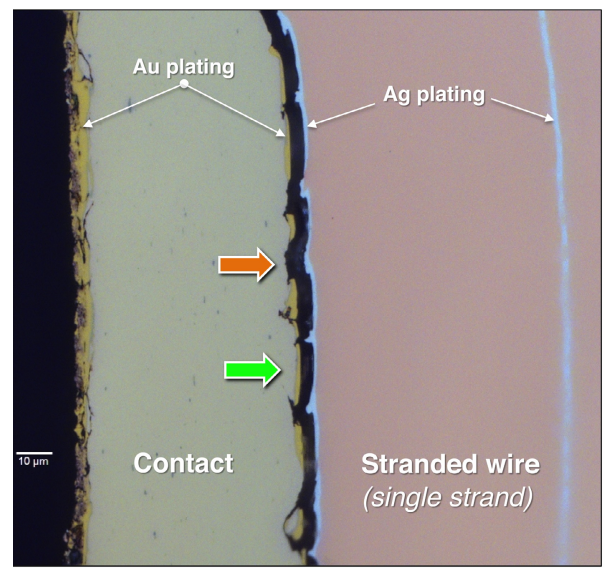

(b)

Fig. 1 (a) Optical micrographs shows the absence of a bond (red arrows). (b) The green and orange arrows indicate $\mathrm{Au} / \mathrm{Ag}$ interface failure and bare $\mathrm{Be}-\mathrm{Cu} / \mathrm{Ag}$ interface failure, respectively.

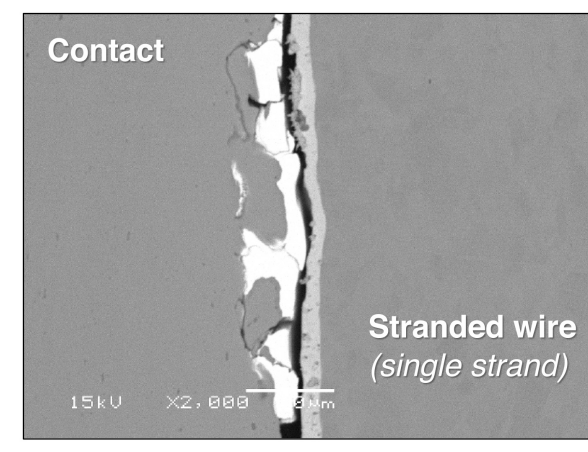

(a)

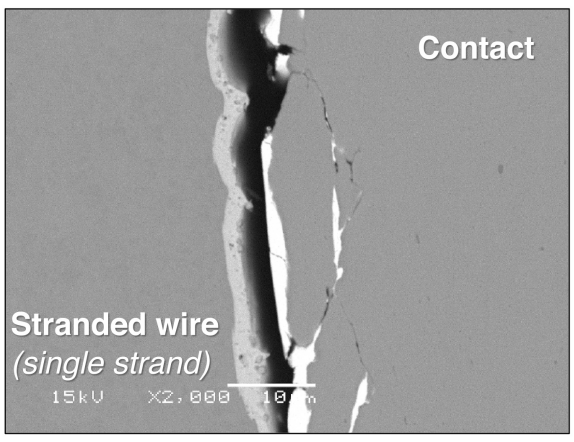

(b)

Fig. 2 SEM photographs show the damage to the contact material surface and sub-surface regions as indicated by by the electroplating of $\mathrm{Au}$ within the cracks and gaps. 